$\mathbf{N}$

O

T

A

S 


\title{
LA RACIONALIDAD EN EL PENSAMIENTO Y EN LA ACCIÓN
}

\author{
Matti Sintonen \\ Universidad de Tampere
}

\section{El hombre, ¿un animal racional?}

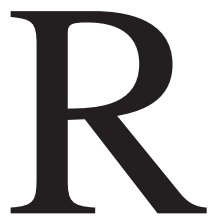

ecuerdo la siguiente discusión durante un desayuno en Berlín. Frente a mí se sentaba un hombre que se dedicaba a supervisar la labor de una de las compañías más grandes del mundo (por el número de empleados), la East-German Treuhand. Al enterarse de que yo era un filósofo que por profesión creía en la razón y el progreso, comentó que él creía en el progreso, pero no en la razón. Los filósofos dan por sentado que el hombre es un ser racional, pero él tenía razones para creer otra cosa. Se ocupaba de tratar de racionalizar una empresa que podría no sobrevivir a la apertura a la feroz competencia occidental de un sector antes cerrado. Esto se llama racionalización, y su objetivo era vender fábricas y servicios. Ciertamente, esto amenazaba con hacer redundantes muchos empleos; pero, por otro lado, también prometía asegurar el trabajo de muchas otras personas. Sin embargo, cada vez que presentaba a una fábrica una propuesta, ésta era objetada con pretextos concretos que trataban de mostrar que, en este caso, en realidad, no era necesario llevarla a la práctica. Todo el mundo admitía que había que hacer algo. Pero de la misma manera cada uno tenía razones concretas por las que ese trabajo en particular no era preciso.

Aquí se plantean importantes cuestiones. En primer lugar, por qué la racionalidad es una noción relevante; en segundo lugar, antes de poder profundizar en la cuestión de si el hombre es un animal racional, debemos entender bien qué es ser racional. En tercer lugar, podemos preguntarnos en qué ámbito se sitúa la noción de racionalidad: normalmente pensamos que un individuo es racional o irracional, pero los empresarios creen más bien que es el mercado impersonal el que eventualmente dicta qué es lo racional. La mano invisible guía no sólo a las sociedades, sino también a la naturaleza: los pájaros no son a nuestros ojos los agentes más racionales, pero in- 
cluso su conducta extraña se aproxima al óptimo. Finalmente, hay cierto número de cuestiones que las ciencias humanas deben encarar. Una de ellas es la cuestión de hasta qué punto somos racionales -y hasta qué punto podemos ser estúpidos y seguir siendo considerados como agentes-. Otra es la cuestión de si existe una noción de racionalidad única, universal y, a la vez, interesante.

\section{Algunas cuestiones sobre la racionalidad}

Quizá es más fácil comenzar con las cuestiones acerca de qué lugar ocupan la razón y la racionalidad en el inventario del mundo y por qué estas nociones son importantes. Es obvio que la noción de racionalidad derivada de nuestra facultad de razón y su uso en la formación de creencias y como guía para la acción es esencial en nuestra visión del mundo. En efecto, la concepción tradicional es que lo característico de los hombres reside en su capacidad para la deliberación racional acerca de qué aceptar como verdadero, o verosímil, o adecuado, qué asumir como fines y qué hacer en determinadas circunstancias.

Decir que el hombre es un ser racional implica también afirmar que es capaz de planear su futuro, y esto parece ser exclusivamente humano. El hombre es el único animal con una mente o alma estructurada de manera que las leyes superiores de la razón y el entendimiento limitan la satisfacción de los instintos y deseos inferiores, esto es, de los instintos animales. Esto no debería tomarse como un axioma de la antropología, porque una razón flexible, en principio, es posible para los miembros de otras especies (tales como artefactos o marcianos). De la misma manera, no debería implicar que todo el mundo es racional todo el tiempo, ni siquiera que la mayoría de la gente es racional la mayoría del tiempo. Más bien, ya sea una capacidad o una propiedad disposicional, la racionalidad pertenece a la manera como normalmente nos describimos a nosotros mismos o a otros (pertenece al aparato conceptual usado para describir las acciones humanas).

La racionalidad no es sólo una noción profundamente enraizada en nuestra imagen de nosotros mismos, sino que también constituye un importante principio en las ciencias humanas. Este principio -denominado el Principio de Racionalidad- opera no sólo en filosofía (incluyendo la ética y la filosofía social), sino también en psicología, sociología, antropología y algunos sectores de la arqueología, historia, educación, literatura y otras áreas de las humanidades y de las ciencias sociales. Y, por supuesto, las ciencias sociales, en el sentido relevante, incluyen también las ciencias jurídicas. Uno de los debates en la filosofía de las ciencias sociales de este siglo es el estatus de este principio. ¿Es una observación empírica o una generalización sobre los miembros del homo sapiens? ¿O es un principio me- 
todológico que guía toda interpretación y formación de hipótesis en las ciencias del hombre? ¿O un principio normativo o a priori que constituye una condición para percibir a los demás como seres humanos, o para la posibilidad de una ciencia del hombre?

El relato que he presentado al comienzo muestra también que la racionalidad es a la vez importante para la teoría y para la práctica. No es sólo una clave central en el entramado de conceptos que usamos para describir y explicar la conducta individual y las instituciones sociales, sino una noción con una dimensión práctica. Una de las formas en las que esto se manifiesta es en el conflicto potencial entre la racionalidad individual y la colectiva: existe un paradigma de racionalidad individual, que discutiré más adelante, en el que la racionalidad se refiere a acciones (en concreto, decisiones) que conducen a la máxima satisfacción de los deseos del agente. Pero hay ejemplos -como el dilema de los prisioneros- en los que los individuos racionales que persiguen sus objetivos no pueden conseguir el resultado óptimo (el mejor para todos). El dilema de los prisioneros y otros problemas de racionalidad colectiva son teóricamente interesantes porque nos conducen al siguiente rompecabezas: ¿Cómo son posibles los patrones de conducta cooperativos y desinteresados? Si perseguimos nuestros fines individuales y maximizar nuestro bienestar (cualquiera que sea su definición), la conducta egoísta y parasitaria debería constituir la norma. ¿Cómo es posible, entonces, el altruismo? Un análisis de las condiciones en las que este dilema se plantea es de una enorme relevancia práctica para la teoría económica, la política social y, por supuesto, para las políticas internacionales y el Derecho. Basta recordar el reciente encuentro de alto nivel sobre política medioambiental en Niza para persuadirse de que es necesario ser capaces de identificar y evitar situaciones en las que la racionalidad individual y el interés colectivo entran en conflicto. En este encuentro, cuidadosamente preparado, todos los estados y representantes estaban de acuerdo en que combatir el efecto invernadero llevaría a un resultado que sería mejor para todos. Pero, por razones que vale la pena analizar usando la teoría de los juegos y modelos de decisión, podría tomarse una decisión distinta a la que intuitivamente sería la mejor y más racional.

\section{Razón teórica y razón práctica}

Una vez vistas estas cuestiones sobre la racionalidad, la siguiente pregunta es qué es la racionalidad. Esta es una cuestión calurosamente debatida en la filosofía occidental. La noción de racionalidad -en la medida en que haya sólo una noción- puede ser enfocada desde una perspectiva normativa, descriptiva o explicativa. La racionalidad, como noción normativa, trata de mostrar lo que debería creer, hacer, o qué fines debería tener, deter- 
minado sujeto en una particular situación epistémica o de otro tipo. Decir que la racionalidad es una noción normativa implica que tal noción debería proyectarse sobre las contingencias psicológicas o sociales con tanta claridad como se aplica al pensamiento y a las acciones individuales. Como noción descriptiva nos lleva a describir cómo los sujetos forman de hecho sus creencias y seleccionan sus fines y acciones. Como noción explicativa se usa para explicar por qué los individuos tienen las creencias que tienen, o los fines que tienen, o por qué hacen las acciones que hacen. Un problema, que será discutido brevemente más adelante, es si las nociones normativas usadas normalmente tienen una importancia descriptiva o explicativa. Debería señalarse también que los conceptos y leyes que describen la conducta podrían no ser explicativas, y viceversa. Nancy Cartwright (1983) ha sugerido que las leyes de la física son falsas en el siguiente sentido: las leyes sobre fenómenos que son verdaderas de los fenómenos naturales son descriptivas pero no explican. Sin embargo, las leyes fundamentales de la naturaleza que utilizan construcciones teóricas y proveen de explicaciones son demasiado abstractas para ser descripciones verdaderas de regularidades percibidas. La misma posibilidad se plantea también, al menos en principio, para las ciencias del hombre.

La racionalidad es una noción moldeada históricamente y quizá pueda entenderse mejor con ayuda de una serie de distinciones. Esta noción es usada con sentidos distintos en la vida y el lenguaje cotidianos, y los análisis filosóficos normalmente toman uno de esos sentidos para un análisis más detallado. En lo que sigue adoptaré y adaptaré la lista de Stephen Luke's (1979) de posibles fallos de la racionalidad (esta lista no es la única presentada por Lukes; para una lista ligeramente distinta véase, por ejemplo, Elster, 1982). De acuerdo con Lukes, una creencia (o un conjunto de creencias) debe ser considerada irracional si fracasa en alguno o todos los siguientes requisitos: En primer lugar, un conjunto de creencias puede ser inconsistente o ilógico, y en ese sentido irracional. En segundo lugar, miembros de un conjunto de creencias pueden ser erróneos en todo o en parte y, en tercer lugar, insanos en algún sentido. En cuarto lugar, una creencia o grupo de creencias puede ser ad hoc y, como dicen los filósofos de la ciencia, estar dirigidas a alguna situación estrecha. Tales creencias no pueden ser generalizaciones y no existe justificación independiente adicional para sostenerlas. En quinto lugar, las creencias pueden ser irracionales en el sentido de que pueden estar basadas en premisas irrelevantes en todo o en parte, o derivadas de o basadas en pruebas insuficientes, o ser adoptadas acríticamente sin ser sometidas al test empírico y de observación. En el caso extremo, una creencia empírica o no lógica y no analítica se podría considerar sagrada o más allá de la crítica para defenderla frente a la evidencia con- 
traria. Además, en esta categoría de creencias irracionales podemos incluir aquellas que son adoptadas sin reflexión y sin prestar atención a las presuposiciones e implicaciones subyacentes a ellas o vinculadas con sus consecuencias.

Además de éstos, Lukes también señala otros usos de la palabra racionalidad, tales como, en sexto lugar, la perspectiva que equipara racionalidad con acción dirigida a fines, o, en sexto lugar, la acción que, desde el punto de vista del agente, es el mejor medio para conseguir un fin determinado. Además, en octavo lugar, la racionalidad también puede referirse a acciones con las que una persona adopta de hecho (y no sólo según su propia opinión) los mejores medios posibles para alcanzar un fin, y, en noveno lugar, la acción que de hecho y de manera continuada se dirige hacia un fin. Finalmente, en décimo lugar, la racionalidad puede referirse a fines del agente, en el sentido de que un agente racional sólo tiene fines razonables. Esta es la noción de objetivo o fin racional. Las distinciones de Lukes, por tanto, dan cuenta de tres de las más importantes áreas de aplicación de la noción de racionalidad, esto es, el pensamiento, la acción y los fines, donde la última está claramente relacionada con la segunda.

Este análisis tripartito de la racionalidad de creencias, medios y fines, se refiere a dos tipos diferentes de razón, teórica y práctica, o dos tipos diferentes de facultades. La formación de creencias, a su vez, puede referirse a creencias dentro del ámbito de los meros sentidos o -de dos maneras distintas- a creencias que van más allá de ellos. Por un lado, están las creencias que se basan en la aprehensión por medio de la percepción y un aparato conceptual cuyas generalizaciones van más allá de las observaciones singulares: son generalizaciones inductivas. Por otro lado, están las creencias más teóricas, que contienen referencias a entidades inobservables o construcciones teóricas. Así, llegamos al terreno de la epistemología de las creencias perceptuales, la lógica de la inferencia inductiva y la lógica de la inferencia abductiva, en el sentido de Pierce y Hanson.

La razón teórica tiene una larga historia en la filosofía occidental, desde Platón y Aristóteles hasta los pragmatistas y constructivistas de nuestros días, pasando por los racionalistas y empiristas. No hay una caracterización universalmente aceptada de la naturaleza y el ámbito de la razón teórica. Lo que puede decirse en todo caso es que su relación con otras facultades, especialmente con las capacidades o facultades cognitiva, impulsiva y emotiva, ha sido un tópico constante de debate a lo largo de los siglos. La opinión más ampliamente aceptada es que hemos estado viviendo en la época de la razón desde el desarrollo de la identidad moral alrededor del temprano Renacimiento. Esto significa que la razón, y especialmente la razón teórica, ha sido la característica definitoria de la esencia del hombre. 
La razón práctica, por su parte, tiene que ver con los objetivos de las acciones y los medios para alcanzarlos. Una tradición filosófica ha sostenido, desde David Hume, que la deliberación práctica se limita a la elección de medios -así, la razón práctica es modelada sobre la deliberación teórica. De esta manera, lo que el razonamiento puede hacer es calcular, una vez que las pasiones y los deseos han señalado un fin y motivado un propósito, cuál es el mejor medio para llegar a ese objetivo. Un buen ejemplo de esta noción es la teoría de la elección racional de la moderna teoría económica. Esta ha alcanzado el más alto refinamiento de la razón o racionalidad instrumental, un particular enfoque de esta tradición en el que la razón teórica invade el ámbito de la práctica. Aquí normalmente se toma el pretexto de maximizar los beneficios o algún otro objetivo. Por supuesto, esta visión de la racionalidad instrumental ha sido sometida a duras críticas por escritores como Horkheimer y Adorno (1972), Charles Taylor (1989), Alisdair McIntyre (1988) y Stephen Toulmin (1990). No sólo ha sido criticada la reducción de la racionalidad al aspecto técnico, instrumental y estratégico (social); muchos autores creen que los valores comunitariamente compartidos determinan en gran medida nuestros fines y que, consiguientemente, las consideraciones morales y sociales son incluso más importantes para la identidad moderna que los principios epistémicos de la racionalidad. Esto no implicaría que la razón o la racionalidad tuviera que dejar paso a la moralidad. Más bien implicaría que esas nociones estarían impregnadas de un aroma moral. Desafortunadamente, ahora no puedo profundizar más en esta discusión acerca de la racionalidad moderna.

\section{Individualismo, holismo y culturalismo}

Son pertinentes aquí un par de matizaciones sobre las variedades de irracionalidad presentadas por Lukes. Primero, las bases sobre las cuales se atribuye irracionalidad a las creencias o, más exactamente, sobre las que un agente adopta tales creencias, varían de caso a caso. La distinción más importante es la distinción entre la justificación de los contenidos de las creencias y la justificación de la manera como el agente se ha formado tales creencias. En los casos 1-3 (y posiblemente 4) las creencias son incoherentes, extrañas o algo semejante, mientras que el caso 5 se centra en el razonamiento detrás de la creencia. La distinción es importante, aunque podemos preguntarnos si sostener una determinada creencia puede juzgarse como irracional sin tomar en cuenta el modo como se ha llegado a ella. Por esta razón, es también claro que no todas las creencias falsas son irracionales: pueden ser el resultado de fallos en el funcionamiento del aparato de percepción o en el razonamiento. Todos los agentes racionales tienen cre- 
encias falsas -que parecen tener algún tipo de coherencia con el resto de creencias que el agente tiene razón en aceptar-.

Otra distinción es relativa a la epistemología y metafísica de la adscripción de creencias. ¿Es posible evaluar creencias individuales? Los individualistas acerca de las creencias contestarían positivamente a esta cuestión, mientras que los «molecularistas» y holistas dirían, siguiendo a Quine (1960), que las creencias no se someten aisladamente al tribunal de la experiencia sensorial, sino como un cuerpo conjunto. Esta línea de pensamiento puede llevarse más lejos todavía: Donald Davidson (1973) y muchos otros han defendido que sólo puede adscribirse una creencia con un contenido proposicional específico a un agente si tiene un gran número de otras creencias (¡y, además, correctas!). Imaginemos: ¿Sería posible para alguien tener una única creencia, por ejemplo que Alicante está al sur de Barcelona?

Una tercera distinción tiene que ver con diferentes tipos de individualismo y holismo. Ya hemos visto la cuestión acerca del nivel de racionalidad. Pero el debate acerca de la racionalidad «primitiva» y los experimentos mentales sobre la posibilidad de extraños tipos de racionalidad, plantean el problema de qué niveles de racionalidad pueden ser juzgados. Hemos asumido antes que los agentes individuales están cargados de creencias. Pero sabemos por la experiencia y la historia que han sido considerados como racionales clases diferentes de creencias y, si la historia enseña algo, es seguramente que no estamos en este asunto en mejor situación que nuestros predecesores. Y si las creencias se someten al tribunal de la experiencia sólo como un todo y la mayoría de nuestras creencias han sido adoptadas más bien acríticamente, ¿qué justificación tenemos para considerar irracionales las creencias de alguien?

Ahora podemos replantearnos la distinción entre creencias y razonamiento, y las normas de razonamiento en la formación de creencias. Claramente, un nivel de juicio más compartido podría estar constituido por las normas epistémicas generalmente aceptadas. Así, un agente sería irracional si él o ella falla al formar creencias sin las precauciones epistemológicas y científicas necesarias. Sin embargo, el problema que surge ahora es que también las normas epistémicas y metodológicas cambian; así que ¿sería posible evaluar los cánones de siglos anteriores como racionales o irracionales? Y, si fuera así, ¿con qué estándares? ¿Hay una piedra de toque para la racionalidad que pueda ser usada como una norma epistémica intercultural? A quienes defienden algún tipo de visión científica del mundo les gustaría contestar que sí: puede que no estemos en el mejor de los mundos metodológicamente posibles, pero nuestras prácticas científicas se aproximarán (o deberían hacerlo) a las prescritas por El Método. Si existe tal método (o si, por el contrario, casi todo vale), es, por supuesto, una de los pun- 
tos más apasionadamente discutidos en la filosofía de la ciencia. Y si nosotros poseemos El Método, ¿tenemos el deber moral de difundirlo y enseñárselo a aquellos que todavía no lo conocen?

La distinción es también aplicable al nivel de la razón práctica. Una acción individual podría ser considerada irracional si no reúne los requisitos detallados en la lista de Lukes; por ejemplo, si los medios escogidos para conseguir el objetivo deseado no fueran (de hecho o desde la perspectiva de las creencias de los agentes) óptimos o fueran autofrustrantes. Y una elección podría ser considerada irracional si no se ajustara al modelo de los economistas de la maximización de la utilidad esperada. Pero, de nuevo, el acento podría ponerse en el segundo nivel, el de los estándares aceptados de racionalidad: las razones para la acción se encuentran determinadas por la comunidad, y acciones que en una cultura pueden ser consideradas absurdas o ridículas o irracionales, pueden ser plenamente aceptadas en otras. De manera que la racionalidad quizá podría ser relativizada a una determinada cultura en un momento concreto. ¿Pero sería posible someter a tales normas a una valoración crítica? La mera existencia de facto de tipos diferentes de normas morales en distintas sociedades no permiten reivindicar el relativismo moral en ningún sentido fuerte, así que el hecho de que algunos patrones de comportamiento sean aceptados en algunas culturas no implica que no puedan ser criticados desde el punto de vista de alguna normas universales.

Si existe progreso en el mejoramiento de los estándares de prudencia y juicios morales es otra cuestión perenne de la filosofía. En cualquier caso, está claro que podemos plantear cuestiones de racionalidad práctica en el ámbito de la acción teórica y en el de las normas e instituciones que impulsan y motivan la acción. Así, nos encontramos con diferentes tipos de teoría de la acción en función del esquema que se adopte para la descripción y explicación de la conducta (tal como la perspectiva intencionalista y la causalista). Y, por supuesto, tenemos varios tipos de teorías de la acción social en función del esquema adoptado para la descripción y explicación de la acción colectiva. Por último, nos encontramos con una u otra filosofía moral y social, incluyendo la filosofía política y jurídica, en función de qué crítica social se haga y de qué discurso sobre la racionalidad de las normas y prácticas se asuma.

\section{MINRAT y el método: ¿Hasta qué punto podemos ser estúpidos (y seguir contando como agente)?}

El concepto instrumental de racionalidad parece ser una principio general que guía toda formación de conceptos y teorías, también el las ciencias sociales y humanas. A menudo se conoce a esto como el Principio de Ra- 
cionalidad, de acuerdo con el cual un agente es racional sí y sólo si elige los medios que, de hecho o desde la perspectiva de las creencias, produce, o es adecuado para producir, sus fines.

Pero ¿hasta qué punto somos racionales? Las nociones normativas de creencia racional y de acción instrumental racional, tal como encuentran expresión en el modelo del hombre económico racional, son extremadamente exigentes. Suponen que un agente racional suscribe una lógica (de primer orden) completa y correcta, y consiguientemente no sólo cree en un conjunto de axiomas de un sistema, sino también en todas sus consecuencias. Un agente racional ideal, además, siempre elegiría la acción que maximiza sus objetivos. Como Christopher Cherniak $(1986,7)$ señaló: «Si A tiene una creencias más un deseo determinado, A emprendería todas las acciones que son aparentemente apropiadas, y sólo ellas».

Cherniak cree que no puede exigirse una concepción como ésta de la racionalidad ideal. Presenta el inconveniente de que es psicológicamente irreal y por ello incapaz de explicar la conducta real. Nadie cree en todas las consecuencias de su propio sistema de creencias (sabemos que esto es imposible; véase Tvesrky y Kahneman, 1974), y todo sistema real de creencias contiene contradicciones y lagunas. Además, la noción idealizada de racionalidad instrumental falla en dar cuenta de los resultados empíricos, que muestran claramente que las preferencias de las personas no son siempre consistentes, y que la gente comete lo que los estadísticos llaman falacias (tales como mantener que una conjunción es más probable que uno de los miembros de la misma). A esto hay que añadir que esas falacias son virtualmente independientes de la educación: tendemos a razonar incorrectamente, sin considerar el entrenamiento formal que tengamos. Se nos debe enseñar a identificar patrones incorrectos de razonamiento.

La conclusión de Cherniak es que, en lugar de una noción ideal de racionalidad que nadie puede cumplir, necesitamos una noción de racionalidad mínima [minimal rationality] o MINRAT. La gente razona de acuerdo con situaciones contextuales y enfocan lo que destaca y es importante aquí y ahora. En lugar de perder tiempo y recursos calculando el resultado óptimo, la gente queda satisfecha simplemente tomando la primera opción que funcione razonablemente bien. Así, Cherniak prima al sentido común y a lo razonable sobre los criterios formales: dado un tiempo limitado y recursos útiles podría ser irracional perder todo el tiempo calculando cuál sería la mejor decisión. La racionalidad mínima, dice, tiene la característica de que la mayoría de nosotros puede ser racional en ese sentido.

Una de las consecuencias de esta perspectiva es que necesitamos preguntarnos hasta qué punto puede debilitarse el estándar. El motivo es éste: Para una concepción atractiva, la racionalidad es un rasgo constitutivo de la 
personalidad o de la calidad de agente. Esta es precisamente la razón por la que la noción es importante. Si las creencias y deseos de alguien no producen lo que a nuestro juicio son las acciones razonablemente adecuadas -si no siempre, al menos en la mayoría de ocasiones-, tendemos a pensar que hay algo seriamente defectuoso en la arquitectura mental de ese sujeto. Y si sus deseos y creencias son totalmente alocados y parecen tener poca conexión con la acción, llegamos a la conclusión de no atribuirle calidad de agente en absoluto: ¡sin racionalidad, no hay agente! (véase Dennett, 1987). Es por esto por lo que debenos preguntarnos hasta qué punto puede ser debilitada la frontera: ¿hasta qué punto se puede ser estúpido y seguir contando como agente?

\section{La racionalidad universal y la brujería}

La última cuestión que deseo tratar es la noción de universalidad. En la concepción de Kant, tanto la racionalidad teórica como la práctica son universales y suprahistóricas. Así, la razón teórica no es sólo la razón del hombre occidental educado, sino, en principio, algo que sobrepasa culturas, razas, géneros e incluso las fronteras entre las especies. Esto significa que cualquier organismo u otra entidad que reúna ciertos requisitos normativos podría ser adecuado. De la misma manera, el hombre es un agente moralmente responsable que es libre de escoger entre cursos de acción alternativos. Pero las exigencias de las razones prácticas requieren que la moral del agente sea -en la medida en que se trata de un agente moral- conforme con el imperativo categórico y con las máximas más detalladas.

Esta asunción de racionalidad universal ha sido discutida a la luz de la evidencia antropológica. Así, las distintas escuelas o variedades de relativismo han dudado de la existencia de una serie de principios de racionalidad que sean a la vez universales e interesantes. Nosotros tendemos a creer que nuestras normas son racionales, y tendemos a juzgar con ellas a otras normas propias de otras culturas. ¿No será esto sólo chovinismo?.

Lo que sigue se refiere sólo a la creencia racional. En antropología hubo un debate sobre la racionalidad en el que se discutió la posibilidad de evaluar las prácticas culturales de otra cultura. E.E. Evans-Pritchard argumentó que el razonamiendo de los Azande no parece ajustarse a los estándares de la lógica y la racionalidad, tal como se han manifestado en la ciencia y la tecnología occidental. Dicho a grandes rasgos: aunque los Azande sostienen creencias que ellos mismos perciben como inconsistentes o defectuosas al acomodarlas unas a otras, se niegan a derivar la conclusión lógicamente inevitable.

El ejemplo del pensamiento Azande que Evans-Prichard considera tiene que ver con la brujería. Los Azande creen que todos los hechiceros tienen 
una sustancia mágica en sus vientres que puede ser descubierta con un examen post-mortem. Asimismo, creen que la sustancia mágica se hereda por línea masculina, de modo que todos $-\mathrm{y}$ sólo ellos- los hijos de brujos tienen la misma sustancia y, por consiguiente, son brujos. Sin embargo, los Azande también saben que todos los clanes se componen de individuos relacionados por línea masculina. Y este conjunto de creencias crea un problema. Como escribe Evans-Pritchard: «A nuestra mente le parece evidente que si se prueba que un hombre es un brujo, entonces todos los miembros de su clan son ipso facto brujos, dado que el clan Azande es un grupo de personas relacionadas biológicamente unas con otras a través de la línea masculina». Sin embargo, la cuestión aparece planteada de forma diferente en la mente de los Azande: ellos se niegan a aceptar la conclusión precisamente porque no es posible que todos los miembros de un clan sean brujos.

Evans-Pritchard piensa que los Azande en realidad «ven el sentido del argumento», pero en lugar de abandonar algunas de sus creencias prefieren emplear estrategias que les permiten vivir con la contradicción. También ofrece una explicación parcial de este hecho: los Azande no ven sus contradicciones tal como nosotros las vemos «porque no tienen un interés teórico en la cuestión». Las «situaciones en las que ellos expresan sus creencias acerca de la brujería no les plantean ningún problema».

Aunque Evans-Pritchard comparte con los antropólogos la preocupación por encuadrar la brujería y magia de los Azande con nuestra lógica y racionalidad occidental, Peter Winch (1964) no cree que haya llegado muy lejos. El descubrimiento - piensa Winch- no es suficiente para acusar a los Azande de irracionalidad, porque el contexto en el que operan las creencias sobre la brujería no es el mismo que el contexto en el que se plantea la acusación de contradicción, «el contexto de nuestra cultura científica». Tanto la lógica como la racionalidad son fenómenos culturales. Las ideas sobre la brujería de los Azande no opera en el mismo nivel, ni «constituyen un sistema teórico con el que los Azande traten de obtener una mejor comprensión del mundo». Winch sugiere que «es el europeo, obsesionado por llevar el pensamiento Azande hasta donde naturalmente no puede llegar (a una contradicción) el culpable de la incomprensión, no los Azande. El europeo está cometiendo, en realidad, un error categorial» (Winch, 1964, pág. 93).

Las observaciones de Winch tocan un punto sensible, porque si tiene razón, Evans-Pritchard fue víctima de un chovinismo científico. Winch fue criticado por Martin Hollis, quien calificó la conferencia de Winch a los antropólogos de winchcraft ${ }^{*}$ (Hollis, 1972; véase también Hollis, 1967). Ho-

\footnotetext{
${ }^{*}$ Nota del traductor: Juego de palabras intraducible, que pone de manifiesto la semejanza entre el término witch (bruja) y el apellido Winch, componiendo el término winchcraft en lugar de witchcraft (brujería).
} 
llis sostiene que es imposible descubrir una lógica o una forma de racionalidad radicalmente diferente en el pensamiento primitivo (o de otro tipo), porque nada que se desvíe demasiado de nuestro pensamiento cuenta como tal.

Hollis puede estar en lo cierto al insistir en un mínimo de lógica y racionalidad compartida. Sin embargo, hay una cuestión más profunda que queda escondida en la casi universal condena de la brujería como irracional. Las llamadas culturas primitivas han sobrevivido durante decenas de miles de años sin la ciencia occidental. Igualmente, la razón teórica es caracterizada como el deseo de y los medios para alcanzar una completa y cada vez más detallada concepción verdadera y desinteresada de la naturaleza y la sociedad. La cuestión crucial es la siguiente: incluso en el caso de que estemos en posesión de la razón teórica y consiguientemente maximicemos la utilidad epistémica en forma de teorías científicas y recientemente de teorías de todo, ¿tenemos algún argumento para mostrar que todo el que ha dado un mordisco a la manzana debería continuar? ¿Podemos demostrar, usando argumentos filosóficos o científicos, que la racionalidad -tal como se entiende en ciencia y tecnología- es o debería ser el objetivo de todo agente racional y de toda sociedad organizada racionalmente? ¿Es posible, racional o razonable, admitir que la ciencia occidental es un juego interesante, pero no obstante rehusar a jugarlo?

Como filósofo yo creo en la razón, pero no me parece claro que la racionalidad científica y tecnológica sean las únicas dimensiones con las que el progreso en la razón pueda ser evaluado. Creo que esto es lo que quiere señalar Habermas (1972) cuando arguyó, enérgicamente, que todo conocimiento está impregnado de algún interés (lo que, por supuesto, no quiere decir que Habermas abandone la racionalidad universal überhaupt). Creo que esto es también lo que quiere resaltar Winch: es chovinismo científico creer que la lógica de la ciencia es la medida de todo.

(trad. de Daniel González Lagier)

\section{Referencias}

Cartwright, Nancy, 1983, How the Laws of Physics Lie. Oxford: Oxford University Press.

CherniaK, Christopher, 1986, Minimal Rationality. Cambridge, Massachusetts: The MIT Press.

DAVIDSON, Donald, 1973, «On the Very Idea of a Conceptual Scheme» (reimpreso en D. Davidson, Essays on Truth and Interpretation. Oxford: Oxford University Press, 1984, pp. 183 - 198). 
Dennett, Daniel, 1987, The Intentional Stance, Cambridge, Massachusetts: The MIT Press.

Elster, Jon, 1982, «Rationality,» en G. Floidstad (ed), Contemporary Philosophy. A New Survey. Vol. 2, pp. 111-131. The Hague, Boston, London: Martinus Nijhoff.

HABERMAS, Jürgen, 1972, Knowledge and Human Interests. London: Heinemann.

HoLLIS, Martin, 1967. «The Limits of Irrationality,» European Journal of Sociology. (Reimpreso en Wilson (ed.), Rationality. Oxford: Blackwell, 1970).

HoLLIS, Martin, 1972, «Witchcraft and Winchcraft,» Philosophy of the Social Sciences, 2, pp. 89-103.

HorkHEIMER, Max y Theodor Adorno, 1972, Dialectic of Enlightenment. New York: Seabury Press.

LuKes, Steven, 1979, «Some Problems about Rationality». En B. Wilson (ed.), Rationality. Oxford: Blackwell.

MacInTYRe, Alasdair, 1988, Whose Justice? Which Rationality? Notre Dame: University of Notre Dame Press.

Quine, Willard van Orman, 1960. Word and Object. Cambridge, Massachusetts: The MIT Press.

TAYLOR, Charles, 1989, The Sources of the Self. Cambridge: The University Press.

TVERsKy, A. y Kahneman, D., 1974, Judgment Under Uncertainty. Science 185, pp. 1124-1130.

WiLson, Bryan, 1970. Rationality. Oxford: Blackwell.

WINCH, Peter, 1964, «Understanding a Primitive Society,» American Philosophical Quarterly 1, pp. 307-24. 
$\triangle \quad$ DOXA 24 (2001) 